\title{
TRANSPORTE DE FÓSFORO E DE POTÁSSIO EM COLUNAS COM AGREGADOS DE UM LATOSSOLO VERMELHO DISTRÓFICO(1)
}

\author{
C. A. S. ARAUJ $0^{(2)}$, H. A. RUIZ(3), P.A.FERREIRA(4), \\ D.J . SILVA ${ }^{(5)}$ \& M. A. CARVALHO(6)
}

\begin{abstract}
RESUMO
O objetivo do trabalho foi comparar os coeficientes dispersivo-difusivos de fósforo e de potássio e descrever o transporte desses nutrientes em diferentes classes de agregados de um Latossolo Vermel ho distrófico, cultivado com mi lho por vários anos, usando dois modelos teóricos. 0 experimento foi realizado, em laboratório, com colunas de percolação, utilizando cinco classes de agregados $(2,0-1,0,1,0-0,5,0,5-0,25,0,25-0,105$ e $<0,105 \mathrm{~mm})$. A eluição foi realizada em um cilindro de vidro de $2 \mathrm{~cm}$ de diâmetro interno e $30 \mathrm{~cm}$ de comprimento, preenchido com agregados até $10 \mathrm{~cm}$ da borda superior. A coluna foi saturada, sob vácuo, com uma solução de $\mathrm{CaCl}_{2} \mathbf{0 , 0 0 5} \mathrm{mol} \mathrm{L}^{-1}$. A velocidade de percolação do efluente foi controlada e mantida próxi ma daquela obtida para a menor classe de agregados. Aplicou-se, a seguir, a solução saturante até percolação constante, seguida de um pulso de uma solução de $\mathrm{KH}_{2} \mathrm{PO}_{4}$ que continha 1.550 e $1.950 \mathrm{mg} \mathrm{L}^{-1}$ de fósfor o e de potássio, respectivamente $\left(\mathrm{C}_{\mathrm{o}}\right)$. No efluente coletado, determi nouse a concentração de fósforo e de potássio (C), que permiti u obter a relação $C / C_{0}$, de acordo com o número de volume de por os da solução percolada. I sso permitiu obter a curva de eluição experimental para esses elementos, a qual foi comparada com curvas teóricas estimadas por dois modelos, um dos quais considera o transporte dispersivo e o outro, o dispersi vo-difusivo. 0 coeficiente dispersivodifusivo para o potássio foi maior do que para o fósforo nas classes de agregados de diâmetro maior que $0,5 \mathrm{~mm}$. Nas de menor diâmetro, ocorreu o contrário, indicando que o fósforo foi transportado mais rapidamente que o potássio nessas colunas de agregados. 0 modelo que considera apenas o fluxo dispersivo apresentou melhor predição de transporte do fósforo e do potássio, em todas as classes de agregados. As curvas teóricas descreveram melhor o transporte de potássio do que o de fósforo.
\end{abstract}

Termos de indexação: transporte de solutos, difusão de fósforo, difusão de potássio.

\footnotetext{
(1) Parte da Tese de Doutorado do primeiro autor junto à Universidade Federal de Viçosa. Recebido para publicação em março de 1999 e aprovado em fevereiro de 2000.

2) Professor do Centro Federal de Educação Tecnológica de Pernambuco (CEFET-PE)/UNED Petrolina, BR 407 km 8, CEP 56300 000 Petrolina (PE).

(3) Professor do Departamento de Solos da Universidade Federal de Viçosa - UFV, CEP 36571-000 Viçosa (MG). Bolsista do CNPq

(4) Professor do Departamento de Engenharia Agrícola, UFV. Bolsista do CNPq.

(5) Pesquisador do Centro de Pesquisa Agropecuária do Trópico Semi-Árido (CPATSA)/ E MBRAPA, CEP $56300-000$ Petrolina (PE).

(6) Professor da Escola Agrotécnica Federal de Colatina, CEP 29700-971 Colatina (ES).
} 


\title{
SUMMARY: PHOSPHORUS AND POTASSIUM TRANSPORT IN COLUMNS WITH AGGREGATES OF A TYPIC HAPLORTOX
}

\begin{abstract}
The objective of this work was to compare the diffusive-dispersive coefficients of phosphorus and potassium as well as to describe nutrient transport in different aggregate classes of a typic Haplortox, cultivated with maize over several years, by applying two theoretical modes. Theexperiment was carried out using percolation col umns and fiveclasses of aggregatesizes (2.0-1.0, 1.0-0.5, 0.5-0.25, 0.25-0.105 and $<0,105 \mathrm{~mm}$ ). The dution col umn consisted of a glass column with $2 \mathrm{~cm}$ internal diameter and $30 \mathrm{~cm}$ length, filled up with aggregates until $10 \mathrm{~cm}$ from the upper boundary. All columns were saturated with a $0.005 \mathrm{~mol} \mathrm{~L}^{-1} \mathrm{CaCl}_{2}$ sol ution, under vacuum. Thevel ocity of the effluent was controll ed and kept near that obtained for thesmallest aggregateclass. Thesaturation solution was applied until reaching a steady flow. Then a slug of a $0.05 \mathrm{~mol} \mathrm{~L}^{-1} \mathrm{KH}_{2} \mathrm{PO}_{4}\left(\mathrm{C}_{0}\right)$ solution was applied. In the collected effluent, concentration of phosphorus and potassium (C) was determined, which permitted to obtain the $\mathrm{C} / \mathrm{C}_{0}$ ratio as a function of the porevolume number of the percol ated sol ution. This all owed to obtain theexperimental curvefor these ements, which was compared with thetheoretical breakthrough curves estimated by thetwo models; oneof them considers only the dispersive transport while the other one considers the diffusive dispersivetransport. Thediffusivedispersive coefficient was higher for potassium than for phosphorus in those classes with greater-diameter aggregates. Theoppositeoccurred in the smallest classes, which indicates that phosphorus moved faster than potassium in those aggregatecol umns, under thestudied conditions. Themode accounting only for thedispersive flow presented a better prediction for phosphorus and potassium transportation in all aggregateclasses. Thetheoretical curves showed a better description for potassi um than for phosphorus transport.
\end{abstract}

Index terms: sol utetransfer, phosphorus diffusion, potassium diffusion.

\section{NTRODUÇÃO}

Desl ocamento miscível é o fenômeno em que um fluido mistura-se com outro e o desloca. Durante o desl ocamento de um fluido por outro, ocorrem vários processos físicos. No instante em que esses dois fluidos se encontram, a interface entre eles é nitidamente definida. Imediatamente após esse contato, inicia-se a mistura entre os fluidos. Essa mistura é influenciada pela velocidade do deslocamento, taxa de difusão do sol uto e por outros processos quími cos efísicos (Niel sen \& Biggar, 1961; Biggar \& Nielsen, 1962; Nielsen \& Biggar, 1963).

No solo, a quase totalidade do deslocamento de soluto ocorre através do espaço interagregado, considerado uma região de sol ução móvel (Passioura, 1971; van Genuchten \& Wierenga, 1976), ondeocorre a dispersão hidrodinâmi ca do soluto, conhecida como dispersão longitudinal, em razão do deslocamento errático dos fluidos no meio poroso (Scheidegger, 1961). Essa forma de dispersão difere da difusão [distribuição de íons devida à sua movimentação ao acaso, considerando sua energia cinética, essencial ao transporte de íons na região de solução imóvel (intra-agregado)], por não depender da concentração do soluto.
É consenso entre vários autores que a dispersão (distribuição) de íons durante um deslocamento de fluidos miscíveis (DM) é devida à dispersão hidrodinâmica, à difusão e aos dois processos ocorrendosimultaneamente (Nielsen \& Biggar, 1961; Biggar \& Nielsen, 1962; van Genuchten \& Wierenga, 1976; Rao et al., 1980).

Vários model os têm si do propostos para descrever o transporte de solutos em meio poroso constituído de agregados. Além da difusão e da dispersão hidrodinâmica, alguns deles consideram outros fatores, tais como: (a) bimodalidade de poros resultante da região interagregados e intraagregados (van Genuchten \& Wierenga, 1976); (b) diferença de concentração do soluto em estudo entre essas duas regiões e o diâmetro equivalente dos agregados (Rao et al., 1982); c) cinética de adsorção-dessorção (Camargo et al., 1979), e (d) distribuição de tamanho dos agregados e do tempo de difusão (Hayot \& Lafolie, 1993).

Segundo Kirkham \& Powers (1972), é conveniente definir os seguintes termos: (a) fluido líquido de composi ção química efísica definida, como, por exemplo, uma solução de nitrato de prata; (b) fluido deslocador - fluido adi cionado para desl ocar um fluido preexistente no meio poroso; c) fluido 
deslocado - fluido que é substituído no meio poroso pel ofluido desl ocador, e (d) efluente- fluido col etado na secção de controle de uma coluna de el uição.

A interface entre dois fluidos no meio poroso não pode ser visualizada. Por isso, considera-se a mudança na concentração do fluido deslocador, no efluente, como a interface entre os dois fluidos emergentes dosol o. A maneira como essa concentração muda fornece informação sobre as características do meio poroso e sobre o comportamento do fluido movendo-se através dele (Nielsen \& Biggar, 1961).

Analisando as mudanças na concentração de ${ }^{36} \mathrm{Cl}$ no efluente de uma col una de sol o, Nielsen \& Biggar (1961) propuseram uma curva-padrão de eluição. Essa curva é obtida relacionando-se $\mathrm{C} / \mathrm{C}_{0}$ em função dep, em queC éa concentração do sol uto no efluente, $\mathrm{C}_{\mathrm{o}}$ a concentração do soluto no fluido desl ocador e $\mathrm{p}$ o número de volume de poros, obtido por:

$$
\mathrm{p}=\mathrm{Qt} / \mathrm{Vo}_{\mathrm{o}}
$$

em que

$\mathrm{Q}=$ vazão do efluente $\left(\mathrm{cm}^{3} \mathrm{~s}^{-1}\right)$;

$\mathrm{t}=$ tempo transcorrido desde a adição do fluido deslocador ao meio (s); e

$\mathrm{V}_{\mathrm{o}}=$ volume de poros da amostra de solo $\left(\mathrm{cm}^{3}\right)$.

A relação $C / C_{0}$, no efluente, é zero no momento da adição do fluido deslocador ao meio, podendo alcançar o valor unitário com o tempo, indicando completa remoção do fluido deslocado. Na maioria dos casos, para íons não-adsorvíveis, essa relação atinge o valor unitário após terem passado dois volumes de poros na coluna de eluição (Nielsen \& Biggar, 1961).

As equações diferenciais que descrevem a dispersão, a difusão ou a interação desses dois mecanismos de desl ocamento deíons no solo, quando resolvidas para condi ções iniciais e de contorno, pelo método analítico ou numérico, permitem quantificar a concentração do soluto no efluente de acordo com o tempo ou o número de volume de poros. As curvas de el uição traçadas com esses val ores denominamse curvas de eluição teóricas. Do ajustamento da curva teórica à experimental, infere-se sobre a validade das pressuposições consideradas para a obtenção e sol ução da equação e sobre a influência no fenômeno real estudado (Kirkham \& Powers, 1972).

$\mathrm{Na}$ interface dos fluidos, um dos mecanismos de mistura pode predominar sobre o outro. Por exemplo, no deslocamento de fluidos miscíveis através de uma coluna curta, de um solo com el evada percentagem de macroporos, sob alta velocidade de avanço, a dispersão predomina sobre a difusão. Em outras situações, predomina a difusão. I sso exige a escol ha correta do modelo físico-matemático, para cada situação a ser estudada, conforme o mecanismo de mistura predominante (Nielsen \& Biggar, 1961, 1963).
Camargo et al . (1979), ao estudarem otransporte de fósforo inorgânico em um Alfissolo (Terra Roxa Estruturada), verificaram que as reações de interação fósforo-agregado, dependentes do tempo, foram reduzidas com a apl icação e incubação prévia de fósforo. I sso permite afirmar que o transporte de fósforo em solos que vêm sendo cultivados e adubados com este elemento, há vários anos, pode ser predito por modelos simples que consideram apenas a dispersão hidrodinâmica e, ou, o coeficiente dispersivo-difusivo do meio.

Considera-se quea quantidade desoluto deslocada por esse mecanismo de transporte pode ser descrita pela Lei do Fluxo Linear sugerida por Kirkham \& Powers (1972), ou seja:

$$
q=-E \frac{\partial C}{\partial x}
$$

em que

$q$ = quantidade do soluto transferida por segundo e por unidade de área perpendicular à direção de percolação $\left(\mathrm{g} \mathrm{cm}^{-2} \mathrm{~s}^{-1}\right)$;

$\mathrm{E}=$ coeficiente de dispersão do soluto $\left(\mathrm{cm}^{2} \mathrm{~s}^{-1}\right)$;

$\mathrm{C}=$ concentração do soluto $\left(\mathrm{g} \mathrm{cm}^{-3}\right)$;

$\mathrm{x}=$ distância $(\mathrm{cm})$.

Taylor (1953), citado por Kirkham \& Powers (1972), descreveu a dispersão longitudinal de solutos pela equação:

$$
\frac{\partial \mathrm{C}}{\partial \mathrm{t}}=\mathrm{E} \frac{\partial^{2} \mathrm{C}}{\partial \mathrm{x}^{2}}
$$

A solução analítica da equação 3 , sujeita às condições iniciais e de contorno

$C(x, t)=C_{0} \quad$ para $x<0$ isto é $-\infty<x<0$ e $t=0$

$C(x, t)=C_{0}$ para $x<0$ e $t=0$

$\lim C(x, t)=0$ para $0<x<\infty$ e $0<t<\infty$ $x \rightarrow \infty$

é

$$
\frac{\mathrm{C}}{\mathrm{C}_{\mathrm{O}}}=\frac{1}{2}\left\{1-\operatorname{erf}\left[\frac{(1-\mathrm{p})}{2[\mathrm{Ep} /(\mathrm{vL})]^{1 / 2}}\right]\right\}
$$

em que

$\mathrm{C}=$ concentração do soluto no efluente $\left(\mathrm{g} \mathrm{cm}^{3}\right)$;

$\mathrm{C}_{\mathrm{o}}=$ concentração do soluto no fluido deslocador $\left(\mathrm{g} \mathrm{cm}^{-3}\right)$;

erf = função erro;

$\mathrm{p}=$ número de volume de poros (adimensional);

$v$ = velocidade média de avanço do efluente na direção x $\left(\mathrm{cm} \mathrm{s}^{-1}\right)$;

$\mathrm{L}=$ comprimento da coluna de solo $(\mathrm{cm})$.

$\mathrm{O}$ coeficiente de dispersão $\mathrm{E}$ é calculado pela relação:

$$
\mathrm{E}=\frac{\mathrm{vL}}{4 \pi \mathrm{S}^{2}}
$$


na qual $\mathrm{S}$ é a declividade da curva experimental, quando $p=1$. A equação 4 é, conforme Kirkham \& Powers (1972), o model o 1 (dispersão).

Na transferência de solutos durante o deslocamento defluidos miscíveis, num meio poroso reativo, com baixa velocidade de avanço, a difusão não pode ser negligenciada (Nielsen \& Biggar, 1962). A equação diferencial, considerando a dispersão e a difusão, conforme Kirkham \& Powers (1972), é

$$
\frac{\partial \mathrm{C}}{\partial \mathrm{t}}=\mathrm{D} \frac{\partial^{2} \mathrm{C}}{\partial \mathrm{x}^{2}}-\mathrm{v} \frac{\partial \mathrm{C}}{\partial \mathrm{x}}
$$

em que

$\mathrm{D}=$ coeficiente dispersivo-difusivo $\left(\mathrm{cm}^{2} \mathrm{~s}^{-1}\right)$.

A solução analítica submetida às condições iniciais e de contorno:

$$
\begin{array}{lll}
C(x, t)=0 & \text { para } x>0 \text { e } & t=0 \\
C(x, t)=C_{0} \quad \text { para } x=0 \text { e } & t>0 \\
\lim _{x \rightarrow \infty} C(x, t)=0 & \text { para } t>0
\end{array}
$$

é o modelo 2, conforme Kirkham \& Powers (1972), ou seja

$$
\frac{\mathrm{C}}{\mathrm{C}_{\mathrm{O}}}=\frac{1}{2}\left\{\operatorname{erfc}\left[\frac{1-\mathrm{p}}{2(\mathrm{Dp} / \mathrm{vL})^{1 / 2}}\right]+\mathrm{e}^{\mathrm{vL} / \mathrm{D}} \operatorname{erfc}\left[\frac{1+\mathrm{p}}{2(\mathrm{Dp} / \mathrm{vL})^{1 / 2}}\right]\right\}
$$

em que

erfc = função-erro complementar.

O coeficiente dispersivo-difusivo éestimado pela relação:

$$
\mathrm{D}=\frac{\mathrm{vL}}{4 \pi \mathrm{S}^{2}}
$$

Conhecendo os valores $\mathrm{V}, \mathrm{L}$ eD para determinado soluto ematerial poroso, podem-se estimar os valores de $C / C_{0}$ para vários val ores de $p$ e construir a curva teórica de eluição. O primeiro termo do segundo membro da equação 7 é igual ao segundo membro da equação 4, para $D=E$. O efeito da difusão é essencialmente estimado pelo segundo termo do segundo membro da equação 7 (modelo 2).

O objetivo deste trabalho foi comparar os coeficientes dispersivo-difusivos do fósforo e do potássio e descrever otransporte desses nutrientes, em diferentes classes de agregados de um $L$ atossolo Vermelho distrófico, usando os modelos 1 e 2 supradescritos.

\section{MATERIAL E MÉTODOS}

Realizou-se, no Laboratório de Análise de Solo e Água do Centro de Pesquisa Agropecuária do Trópico Semi-Árido, EMBRAPA, no município dePetrolina, estado de Pernambuco, um experimento, utilizando colunas de percolação preenchidas com classes de agregados de um Latossolo Vermelho distrófico, cultivado com milho por vários anos. As amostras de solo foram coletadas na camada de $0-5 \mathrm{~cm}$, em quatro locais diferentes, numa área experimental localizada no Centro $\mathrm{N}$ acional de Pesquisa de Milho e Sorgo, EMBRAPA, Sete Lagoas, estado de Minas Gerais.

Após a separação por via seca, adotando o procedimento descrito em EMBRAPA (1979), as classes de agregados de 2,0-1,0, 1,0-0,5, 0,5-0,25, 0,25-0,105 e < 0,105 mm, foram caracterizadas física e quimicamente (Quadro 1).

A mineralogia da fração argila não apresentou variação qualitativa entre as classes de agregados, todas constituídas de caulinita, goethita, hematita, gibbsita eilita.

O experimento, com cada col una preenchida com uma das cinco classes de agregados, com três repetições, num total de 15 colunas, foi instalado para atender às condições iniciais e de contorno dos model os anteriormente citados.

A coluna de percolação, de vidro, com $2 \mathrm{~cm}$ de diâmetro interno e $30 \mathrm{~cm}$ de comprimento, teve a extremidade inferior preenchida com lã de vidro, para evitar perda de material de solo.

As colunas foram preenchidas com os agregados até $10 \mathrm{~cm}$ da borda superior e, a seguir, a superfície foi coberta com lã de vidro, para evitar a destruição dos agr egados pel o impacto do el uente. O volume de poros de cada coluna foi determinado, usando-se a densidade do material seco e empacotado na col una experimental e a densidade das partículas da respectiva classe de agregados (EMBRAPA, 1979).

Fixadas verticalmentenuma estrutura apropriada, as col unas for am submeti das a duas horas de vácuo e, a seguir, saturadas, lentamente, de baixo para cima, com uma solução de $\mathrm{CaCl}_{2}$ 0,005 mol L-1, usando-se um conjunto de alimentação automática (frasco de Mariotte), para diminuir, ao máximo, a presença dear nos poros. Terminada a saturação dos agregados, osistema dealimentação foi posicionado na extremidade superior da coluna, deixando-se percolar a solução de $\mathrm{CaCl}_{2}$, por $16 \mathrm{~h}$. A vel ocidade média de avanço ( $v$ ) da menor classe de agregados (Quadro 2) foi adotada para todo o experimento, sendo controlada, nas outras classes, por meio de uma mangueira, equipada com um regulador de vazão, col ocada na extremidade afunilada da coluna de el uição.

Após atingir o escoamento permanente na coluna de eluição, quando toda a lâmina da solução anterior havia infiltrado, istoé, início do desl ocamento $(t=0)$, aplicou-se um pulso de uma solução de 0,05 mol L-1 de $\mathrm{KH}_{2} \mathrm{PO}_{4}$, correspondente a 1.550 e $1.950 \mathrm{mg} \mathrm{L-1}$ de fósforo e de potássio, respectivamente $\left(\mathrm{C}_{0}\right)$. 
Quadro 1. Caracterização química e física das diferentes classes de agregados de um Latossolo Vermelho distrófico

\begin{tabular}{|c|c|c|c|c|}
\hline \multirow{2}{*}{ Característica } & \multicolumn{4}{|c|}{ Classe de Agregados (mm) } \\
\hline & $2,0-1,0$ & $1,0-0,5$ & $0,5-0,25$ & $0,25-0,105$ \\
\hline pH em água $(1: 2,5)$ & 5,2 & 5,1 & 5,1 & 5,0 \\
\hline $\mathrm{P}$ disponível $\left(\mathrm{mg} \mathrm{dm}^{-3}\right)^{(1)}$ & 5,8 & 6,5 & 7,2 & 8,3 \\
\hline \multicolumn{5}{|l|}{ Complexo sortivo $\left(\mathrm{mmol}_{\mathrm{c}} \mathrm{dm}^{-3}\right)$} \\
\hline$-M g^{(2)}$ & 2,0 & 2,5 & 2,8 & 2,5 \\
\hline - Ca (2) & 7,2 & 7,5 & 8,5 & 9,0 \\
\hline$-K^{(1)}$ & 1,9 & 2,1 & 2,1 & 2,1 \\
\hline$-A l(2)$ & 12,2 & 12,8 & 15,0 & 15,0 \\
\hline$-H^{(3)}$ & 69,6 & 73,4 & 82,5 & 85,5 \\
\hline Carbono orgânico $\left(\mathrm{kg} \mathrm{kg}^{-1}\right)^{(4)}$ & 0,025 & 0,028 & 0,032 & 0,033 \\
\hline \multicolumn{5}{|l|}{ Análise Textural $\left(\mathrm{kg} \mathrm{kg}^{-1}\right)^{(5)}$} \\
\hline - Areia & 0,31 & 0,25 & 0,17 & 0,14 \\
\hline - Silte & 0,16 & 0,20 & 0,27 & 0,24 \\
\hline - Argila & 0,53 & 0,55 & 0,56 & 0,62 \\
\hline \multicolumn{5}{|l|}{ Densidade $\left(\mathrm{kg} \mathrm{dm}^{-3}\right)$} \\
\hline - agregados(6) & 1,48 & 1,61 & 1,42 & 1,39 \\
\hline - partículas(7) & 2,50 & 2,56 & 2,52 & 2,47 \\
\hline
\end{tabular}

(1) Extrator Mehlich-1 (EMBRAPA, 1979). (2) Extrator KCI 1 mol L-1 (EMBRAPA, 1979). ${ }^{(3)}$ Extrator acetato de cálcio (EMBRAPA 1979). ${ }^{(4)}$ Método de Walkley \& Black (J ackson, 1958). ${ }^{(5)}$ Método da pipeta (EMBRAPA, 1979). ${ }^{(6)}$ Método do cimento em pó (Ruiz et al., 1994). ${ }^{(7)}$ Método do balão volumétrico (EMBRAPA, 1979).

Quadro 2. Volume de poros $\left(V_{o}\right)$, velocidade de avanço do efluente $(v)$, declividade da curva de eluição experimental para $C / C_{0}=0,5$ (S), fator de retardamento (R) e coeficiente dispersivo-difusivo (D), para o fósforo e o potássio numa coluna de $20 \mathrm{~cm}$ de agregados, considerando diferentes classes de agregados de um Latossolo Vermelho distrófico

\begin{tabular}{|c|c|c|c|c|c|c|c|c|}
\hline \multirow{2}{*}{ Agregado: } & \multirow{2}{*}{$V_{0}$} & \multirow{2}{*}{$\mathbf{v}^{(1)}$} & \multicolumn{3}{|c|}{ Fósforo } & \multicolumn{3}{|c|}{ Potássio } \\
\hline & & & $\mathbf{S}^{(1)}$ & $\mathbf{R}^{(1)}$ & D & $\mathbf{S}^{(1)}$ & $\mathbf{R}^{(1)}$ & D \\
\hline $\mathrm{mm}$ & $\mathrm{cm}^{3}$ & $\mathrm{~cm} \mathrm{~h}^{-1}$ & & & $\mathrm{~cm}^{2} \mathrm{~h}^{-1}$ & & & $\mathrm{~cm}^{2} \mathrm{~h}^{-1}$ \\
\hline $2,0-1,0$ & 35,8 & 5,107 & 0,148 & 2,925 & 43,37 & 0,155 & 2,069 & 78,89 \\
\hline $1,0-0,5$ & 36,1 & 5,062 & 0,419 & 2,784 & 5,92 & 0,339 & 2,919 & 8,18 \\
\hline $0,5-0,25$ & 35,3 & 5,191 & 0,236 & 3,519 & 11,98 & 0,477 & 3,034 & 3,95 \\
\hline $0,25-0,105$ & 36,4 & 5,511 & 0,254 & 3,354 & 12,10 & nd & nd & nd \\
\hline$<0,105$ & 34,6 & 6,014 & 0,289 & 3,710 & 8,33 & 0,359 & 3,243 & 7,07 \\
\hline
\end{tabular}

(1) Estimado segundo van Genuchten \& Wierenga (1986). nd: Valores não determinados por problemas operacionais.

Cada fração de efluente de aproximadamente 0,25 volume de poros foi coletada e acondicionada em reci pientes plásticos, previamente tarados, com tampas de pressão, e congelada. O volume real do efluente coletado foi determinado pela diferença entre as massas do reci piente com o líquido e vazio. O número de volume de poros (p) foi determinado, dividindo-se o volume acumulado de efluente que passou na coluna de eluição em certo tempo pelo volume de poros da coluna (Quadro 2). Ao final, determinou-sea concentração de fósforo no efluente, colorimetricamente, após formação do complexo fosfomolibdico reduzi do ea concentração de potássio por espectrofotometria de absorção atômica. Conhecendo a concentração de fósforo e de potássio no efluente $(C)$, calculou-sea relação $C / C_{0}$ para cada valor de $p$, traçando-se a curva de eluição experimental. 
As equações 4 e 7 foram obtidas, considerando ser o meio poroso não-reativo. Neste caso, o ponto de inflexão da curva experimental de el ui ção ocorre a $p=1$ volume de poros. Nos meios porosos reativos e, dependendo da espécie de íon presente, há uma tendência natural de a curva de el uição deslocar-se para a direita , isto é, o ponto de inflexão ocorre a $p>1$. Em razão disto, para melhor ajustamento do modelo matemático aos dados experimentais, há necessidade deintroduzir, nos model os, um fator de retardamento ou de adsorção $(R>1)$, e as equações 4 e 7 passam a ser escritas como

$$
\begin{aligned}
& \frac{\mathrm{C}}{\mathrm{C}_{\mathrm{O}}}=\frac{1}{2}\left\{1-\operatorname{erf}\left[\frac{\mathrm{R}-\mathrm{p}}{2[\mathrm{ERp} /(\mathrm{vL})]^{1 / 2}}\right]\right\} \\
& \frac{\mathrm{C}}{\mathrm{C}_{\mathrm{O}}}=\frac{1}{2}\left\{\operatorname{erf}\left[\frac{\mathrm{R}-\mathrm{p}}{2(\mathrm{DRp} / \mathrm{vL})^{1 / 2}}\right]+\mathrm{e}^{\mathrm{vL} / \mathrm{D}} \operatorname{erf}\left[\frac{\mathrm{R}+\mathrm{p}}{2(\mathrm{DRp} / \mathrm{vL})^{1 / 2}}\right]\right\}
\end{aligned}
$$

Pel o fato de o material de sol o utilizado adsorver potássioe, principalmente, fósforo, utilizaram-se, nas simulações, os modelos 9 e 10 e o fator de retardamento $(R)$ foi tomado igual ao número de volume de poros $(p)$, quando $C / C_{0}=0,5$. Para obter o valor do fator de retardamento, foram ajustadas equações de regressão aos dados experimentais, conforme van Genuchten \& Wierenga (1986) (Quadro 2).

Os val ores de $S$ das equações 5 e 8 para fósforo e potássio, para cada classe de agregados, foram obtidos, derivando-se as equações de regressão para val ores de $\mathrm{p}$ correspondentes $\mathrm{a} \mathrm{C} / \mathrm{C}_{0}=0,5$ (Quadro 2 ).

As equações 9 e 10 foram programadas em linguagem basic, para obter as curvas teóricas de eluição.

\section{RESULTADOSE DISCUSSÃO}

A velocidade de escoamento $(v)$ variou entre $5,06 \mathrm{~cm} \mathrm{~h}^{-1}$, nos agregados mai ores, e $6,01 \mathrm{~cm} \mathrm{~h}^{-1}$, nos agregados menores (Quadro 2). A ocorrência de maiores valores de $v$ nas classes de agregados de menor diâmetro justifica-se pelo fato de o escoamento ter sido em meio saturado e vazão constante, regulada a jusante, em todos os ensaios. Como o escoamento em mei o poroso saturado ocorre, essencialmente, nos macroporos, uma mesma vazão passou através de áreas efetivas maiores à medida que o diâmetro dos agregados aumentava.

O coeficiente dispersivo-difusivo (D) diminuiu com o tamanho dos agregados (Quadro 2), em razão da maior adsorção de fósforo e potássio nos agregados menores que, além de retardar o avanço do soluto de concentração $C_{0}(R>1)$, fez com que a declividade (S) das curvas de eluição nos pontos de inflexão aumentasse grandemente, à medi da em que os diâmetros dos agregados diminuíam. Embora os valores de $v$ tenham aumentado nos ensaios com diâmetros de agregados menores, os valores de S aumentaram mais que proporcional aos de $v$ e, conforme a equação 5, os val ores de $\mathrm{D}$ diminuíram.

Fisicamente, este comportamento de $\mathrm{D}$ para agregados menores era, também, esperado, visto que eles contribuem para formação de uma geometria do meio poroso mais uniforme, diminuindo, assim, o caráter errático do movimento junto à frente de avanço $\mathrm{C}_{0}-\mathrm{C}$.

Nas duas classes de agregados de maior tamanho (2,0-1,0 e 1,0-0,5 mm), o modelo 1 , que considera apenas a dispersão hidrodinâmica, apresentou boa predição do movimento do fósforo (Figura 1). Nessas duas classes de agregados, durante o deslocamento da solução na coluna, a interação fósforo-agr egado e a difusão foram insignificantes.

Isto possivel mente deveu-se à menor superfície de contato do fósforo com o solo, isto é, não houve tempo suficiente para o fósforo difundir-se para o interior dos agregados, uma vez que a velocidade de escoamento foi relativamente alta $\left(\geq 5,06 \mathrm{~cm} \mathrm{~h}^{-1}\right)$.

Excetuando a classe de agregados 2,0-1,0, os model os testados, em geral, apresentaram predição aceitável do transporte de fósforo no intervalo de $p$ entre zero e cinco (Figura 1). Além deste intervalo, os valores observados de $C / C_{0}$ foram menores do que os cal culados, mostrando que a concentração $C_{0}$ não foi recuperada, istoé, que o fósforo continuava sendo adsorvido, mesmo após terem sido passados 10 volumes de poros. Tal comportamento foi mais evidente nos ensaios com agregados $\leq 0,5 \mathrm{~mm}$.

Camargo et al. (1979) afirmaram que $90 \%$ das reações em agregados $<1,0$ mm são instantâneas. A interação fósforo-oxiidróxi dos de ferro e dealumínio, minerais constituintes dos agregados estudados, foi bem discutida por Goldberg \& Sposito (1985). Por isso, a vel ocidade de avanço, a qual foi ligeiramente mai or nos agregados menores, não afetou a adsorção. Os resultados indicaram que, mesmo tendo sido adubados com fosfato por vários anos, esses agregados apresentaram ainda alta capacidade de adsorver esse ânion.

Excetuando o ensaio com as classes de agregados 2,0-1,0, o comportamento dos model os 1 e 2 foi semel hante, ou seja, ao adicionar o efeito da difusão para aquela classe de agregados, o modelo superestimou os valores de $\mathrm{C} / \mathrm{C}_{0}$ observados. Esta constatação parece comprovar a quase inexistência de difusão naquela classe de agregados (Figura 1).

As curvas de eluição de fósforo, estimadas pelo modelo 1, o qual apresentou melhor predição, são apresentadas na figura 2. Estas curvas mostram que o desl ocamento para a direita, quando os val ores de 

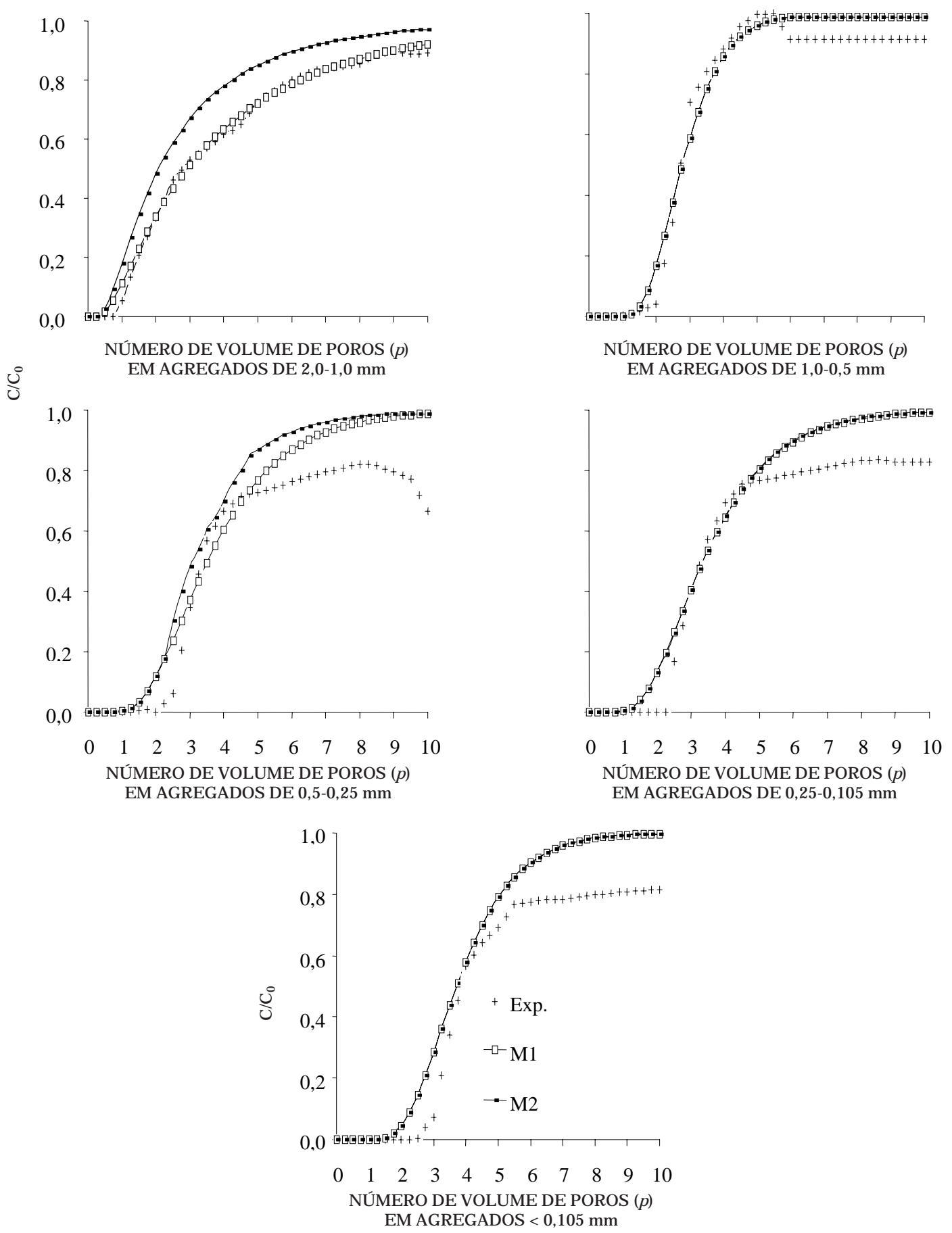

Figura 1. Curvas de eluição para fósforo, experimental (Exp) e calculadas pelos modelos 1 e 2 (M1 e M2), considerando as classes de agregados de um Latossolo Vermelho distrófico.

p estavam entre 0 e 2 , tendiam a aumentar com a redução do tamanho dos agregados, indicando que os agregados mai ores retiveram menor quantidade de fósforo do que os menores no início do deslocamento. Isto evidencia que, por exibirem maior superfície de adsorção, os agregados menores retiveram mais fósforo no início do processo de deslocamento miscível. Tais resultados coincidem com aqueles encontrados por Biggar \& Nielsen (1962) e Rao et al. (1980) que afirmaram que a magnitude do desvio para direita representa a adsorção na superfície dos agregados, a qual está relacionada com o tamanho dos agregados e com sua reatividade. 


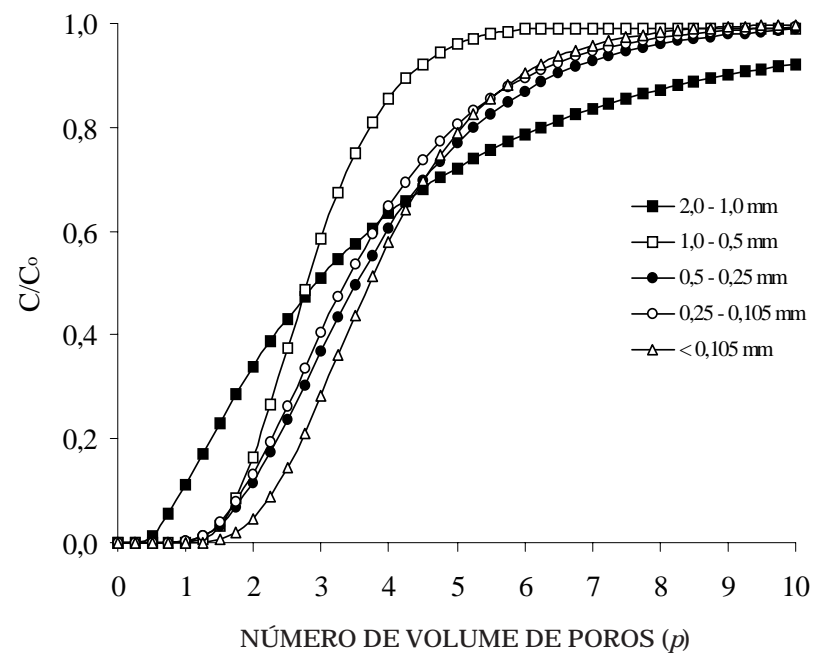

Figura 2. Curvas de elui ção para fósforo, calculadas pelo modelo 1, considerando as classes de agregados de um Latossolo Vermel ho distrófico.
O deslocamento do potássio na coluna de agregados, de maneira geral, assemelhou-se ao do fósforo (Figura 3). Verificaram-se duas diferenças marcantes: o model o 2 só di feriu do model o 1 para a mai or classe deagregados, não se registrando desvio entreas curvas teóricas eas experimentais na parte de declividade menos acentuada das curvas, acima de 4,5 p, como verificado para o fósforo. I sso ocorreu, possivelmente, em razão da baixa capacidade de adsorção catiônica desses materiais, confirmando que a variação entre os valores estimados e experimentais, para ofósforo, resultou da interação fósforo-colóides.

Analisando as curvas estimadas pelo modelo 1 para as diversas classes de agregados (Figura 4), verifica-se que as curvas referentes aos menores agregados também se deslocaram para direita em relação aos dos maiores. I sso resultou da maior área com exposição de sítios de adsorção em relação aos de maior diâmetro.

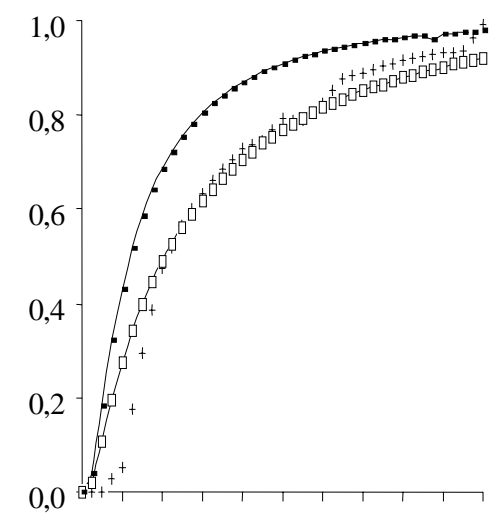

NÚMERO DE VOLUME DE POROS (p) ن

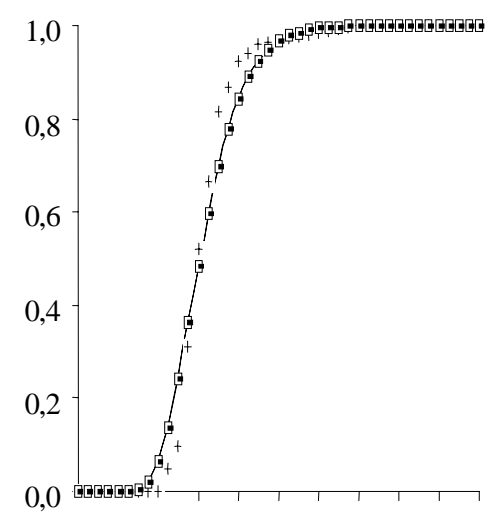

$\begin{array}{llllllllllll}0 & 1 & 2 & 3 & 4 & 5 & 6 & 7 & 8 & 9 & 10\end{array}$

NÚMERO DE VOLUME DE POROS (p) EM AGREGADOS 0,5-0,25 mm

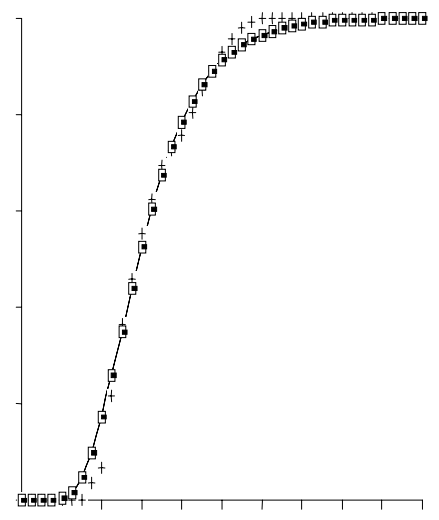

NÚMERO DE VOLUME DE POROS (p) EM AGREGADOS $1,0-0,5 \mathrm{~mm}$

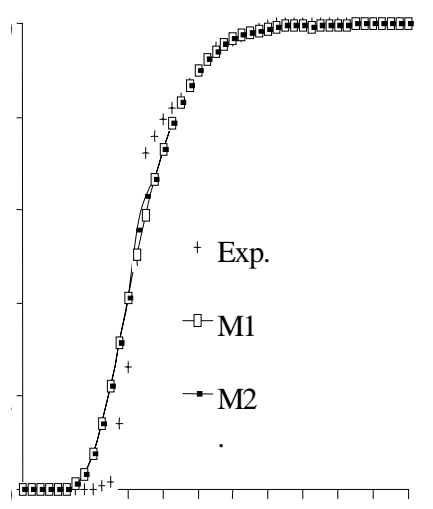

$\begin{array}{llllllllllll}0 & 1 & 2 & 3 & 4 & 5 & 6 & 7 & 8 & 8 & 9 & 10\end{array}$

NÚMERO DE VOLUME DE POROS (p) EM AGREGADOS $<0,105 \mathrm{~mm}$

Figura 3. Curvas de eluição para potássio, experimental (Exp) e calculadas pelos modelos 1 e 2 (M1 e M2), considerando as classes de agregados de um Latossolo Vermelho distrófico. 


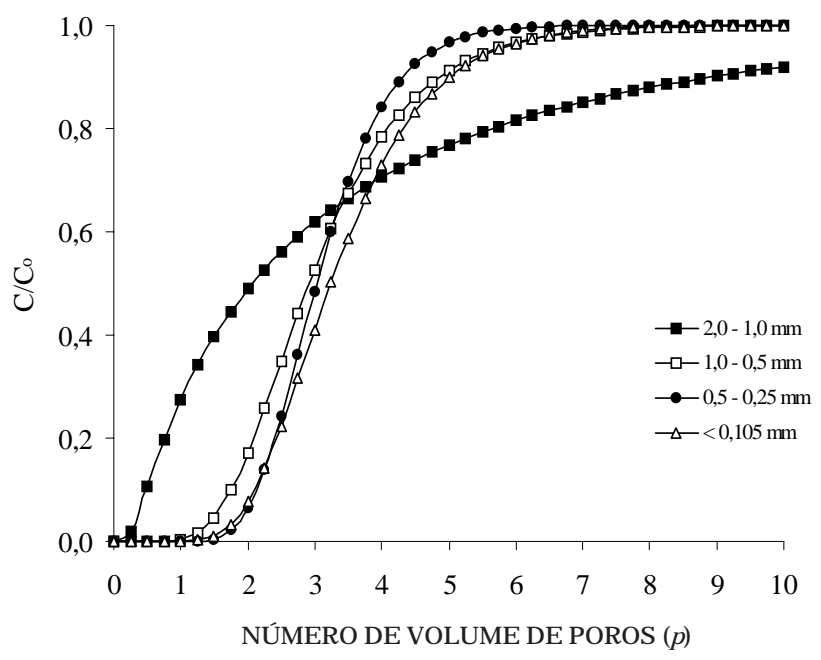

Figura 4. Curvas de el uição para potássio, calculadas pelo modelo 1, considerando as classes de agregados de um Latossolo Vermelho distrófico.

A mobilidade do fósforo e do potássio pode ser comparada pelos valores de D (Quadro 2). Nos agregados de 2,0-1,0, 1,0-0,5 mm, o potássio foi mais móvel que o fósforo, enquanto, naqueles de 0,5-0,25 e $<0,105 \mathrm{~mm}$, ocorreu oinverso, resultante da maior retenção de cátions nessas últimas classes de agregados em relação às primeiras. Como as col unas foram saturadas e submetidas à percolação com solução de $\mathrm{CaCl}_{2} 0,005 \mathrm{~mol} \mathrm{~L}-1$, até atingir o escoamento permanente, o qual ocorreu antes da aplicação da solução 0,05 mol L-1 de $\mathrm{KH}_{2} \mathrm{PO}_{4}$, houve maior adsorção de cálcio nos agregados menores. Durante o deslocamento, o potássio do fluido deslocador foi atraído pelos colóides, deslocando o cál cio, que acompanhou o fosfato, mantendo o sistema eletrostaticamente constante. Akinremi \& Cho (1993) relataram que a mobilidade de K foi retardada com o aumento da capacidade de troca catiônica de uma mistura areia-resina. No estudo desses autores, quando apenas $\mathrm{KH}_{2} \mathrm{PO}_{4}$ foi adicionado na superfície de uma coluna de solo calcário, o fosfato pareceu mover-se à frente do K .

Um estudo feito por Cho (1985), para analisar o transporte de cátions em um sistema de troca de íons, indicou queo Ca desl ocou-se à frente do cátion deslocador $K$. Sabendo que a condição de neutralidade elétrica requer a presença de cargas de íons positivos e negativos em igual número em um vol ume pontual finito, a carga positiva do cátion lixiviado, $\mathrm{Ca}^{2+}$, deve ser satisfeita pela carga negativa do íon fosfato. Conseqüentemente, o fósforo se move à frente do potássio, quando não se adiciona um sal junto com o $\mathrm{KH}_{2} \mathrm{PO}_{4}$.

$\mathrm{Na}$ fertirrigação, recomenda-se aplicar a solução nutritiva, nas últimas horas de aplicação da lâmina de irrigação desejada, de modo que, cessada a aplicação da solução nutritiva, ainda reste uma fração da lâmina deágua por aplicar, suficiente para transportar os nutrientes à profundidade onde se encontra o maior volume de raízes ativas (Coel ho, 1994). Nesse caso, ocorre, verdadeiramente, o desl ocamento de fluidos misć́veis (Nielsen \& Biggar, 1962, Kirkham \& Powers, 1972). A aplicação dessa técnica aos estudos de movimento de íons no solo contribuirá para o uso mais racional da fertirrigação, inclusive utilizando elementos tidos como de baixa mobilidade no solo, como é o caso específico do potássio e, especialmente, do fósforo.

\section{CONCLUSÕES}

1. O coeficiente dispersivo-difusivo para potássio foi maior que para o fósforo nas maiores classes de agregados; nas menores classes, verificou-se o contrário, indicando que o fósforo se moveu mais facilmente que o potássio, em col unas de agregados entre 2,0 e < 0,105 mm, previamente tratados com solução 0,005 mol L-1 de $\mathrm{CaCl}_{2}$.

2. O model o que consider ou apenas o movimento dispersivo apresentou mel hor predição detransporte do fósforo e do potássio, nos agregados entre 2,0 e $<0,105 \mathrm{~mm}$, enquantoas curvas teóricas descreveram mel hor o transporte de potássio do que o de fósforo.

3. Avaliações destetipo têm sido importantes na otimização do manejo e uso de fertilizantes via água de irrigação e recuperação de sol os sal inos, nos quais tipicamente ocorre deslocamento de fluidos miscíveis.

\section{LITE RATURA CITADA}

AKINREMI, O.O. \& CHO, C.M. Phosphorus diffusion retardation in a calcareous system by coapplication of potassium chloride. Soil Sci. Soc. Am. J ., 57:845-850, 1993.

BIGGAR, J.W. \& NIELSEN, D.R. Miscible displacement: II. Behavior of tracers. Soil Sci. Soc. Am. Proc., 26:125-128, 1962.

CAMARGO, O.A.; BIGGAR, J.W. \& NIELSEN, D.R. Transport of inorganic phosphorus in an Alfisol. Soil Sci. Soc. Am. J., 43:884-890, 1979.

CHO, C.M. Ionic transport in soil with ion-exchange reaction. Soil Sci. Soc. Am. J ., 49:1379-1386, 1985.

COELHO, A.M. Fertigação. In: COSTA, E.F.; VIEIRA, R.F. \& VIANA, P.A., eds. Quimigação - aplicação de produtos químicos e biológicos via irrigação. Brasília, Empresa Brasileira de Pesquisa Agropecuária/SPI, 1994. 315p.

EMPRESA BRASILEIRA DE PESQUISA AGROPECUÁRIA EMBRAPA. Manual de métodos de análise de solo. Rio de J aneiro, Serviço Nacional de Levantamento e Conservação de Solos, 1979. não paginado. 
GOLDBERG, S. \& SPOSITO, G. On the mechanism of specific phosphate adsorption by hydroxilated mineral surfaces: a review. Comm. Soil Sci. Plant Anal., 16:801-821, 1985.

HAYOT, C. \& LAFOLIE, F. One-dimensional solute transport modeling in aggregated porous media. Parte 2. Effects of aggregate size distribution. J . Hydrol., 143:85-107, 1993.

J ACKSON, M.L. Soil chemical analysis. New J ersey, Prentice Hall, 1958. 498p.

KIRKHAM, D. \& POWERS, W.L. Advanced soil physics. New York, J ohn Wiley-I nterscience, 1972. 534p.

NIELSEN, D.R. \& BIGGAR, J.W. Miscible displacement: I. Experimental information. Soil Sci. Soc. Am. Proc., 25:1-5, 1961.

NIELSEN, D.R. \& BIGGAR, J.W. Miscible displacement: III. Theoretical considerations. Soil Sci. Soc. Am. Proc., 26:216221, 1962.

NIELSEN, D.R. \& BIGGAR, J.W. Miscible displacement: IV. Mixing in glass beads. Soil Sci. Soc. Am. Proc., 27:10-13, 1963.

PASSI OURA, J .B. Hydrodynamic dispersion in aggregated media. I. Theory. Soil Sci., 111:339-344, 1971.
RAO, P.S.C.; ROLSTON, D.E.;J ESSUP, R.E. \& DAVIDSON,J .M. Solute transport in aggregated porous media: theoretical and experimental evaluation. Soil Sci. Soc. Am. J ., 44:11391146, 1980.

RAO, P.S.C.; J ESSUP, R.E. \& ADDISCOTT, T.M. Experimental and theoretical aspects of solute diffusion in spherical and nonspherical aggregates. Soil Sci., 133:342-349, 1982.

RUIZ, H.A.; ALVES, J .F.O. \& MARTINS, L.G.C. Densidade de agregados separados dos horizontes $A$ e $B$ de um $L V$ e de um PVc. In: REUNIÃO BRASILEIRA DE MANEJ O E CONSERVAÇÃO DO SOLO E DA ÁGUA, 10., Florianópolis, 1994. Anais. Florianópolis, Sociedade Brasileira de Ciência do Solo, 1994. p.332-333.

SCHEIDEGGER, A.E. General theory of dispersion in porous media. J . Geophys. Res., 66:3273-3278, 1961.

van GENUCHTEN, M.T. \& WIERENGA, P.J . Mass transfer studies in sorbing porous media: 1 . Analytical solutions. Soil Sci. Soc. Am. J ., 40:473-480, 1976.

van GENUCHTEN, M.T. \& WIERENGA, P.J . Solute dispersion: coefficients and retardation factors. In: KLUTE, A., ed. Methods of soil analysis. Part 1: physical and mineralogical methods. Madison, American Society of Agronomy, Soil Science Society of American, 1986. p.1025-1031. 